\title{
International severe asthma registry (ISAR): protocol for a global registry
}

J. Mark FitzGerald', Trung N. Tran², Marianna Alacqua ${ }^{2}$, Alan Altraja ${ }^{3}$, Vibeke Backer ${ }^{4}$, Leif Bjermer ${ }^{5}$, Unnur Bjornsdottir ${ }^{6}$, Arnaud Bourdin7, Guy Brusselle ${ }^{8,9}$, Lakmini Bulathsinhala $^{10}$, John Busby ${ }^{11}$, Giorgio W. Canonica ${ }^{12,13}$, Victoria Carter ${ }^{10}$, Isha Chaudhry ${ }^{10}$, You Sook Cho ${ }^{14}$, George Christoff ${ }^{15}$, Borja G. Cosio ${ }^{16}$, Richard W. Costello ${ }^{17}$, Neva Eleangovan ${ }^{10}$, Peter G. Gibson ${ }^{18,19}$, Liam G. Heaney ${ }^{20}$, Enrico Heffler ${ }^{12,13}$, Mark Hew ${ }^{21}$, Naeimeh Hosseini ${ }^{10}$, Takashi Iwanaga ${ }^{22}$, David J. Jackson ${ }^{23}$, Rupert Jones ${ }^{24}$, Mariko S. Koh ${ }^{25}$, Thao Le ${ }^{10}$, Lauri Lehtimäki $^{26}$, Dora Ludviksdottir ${ }^{27}$, Anke H. Maitland-van der Zee ${ }^{28}$, Andrew Menzies-Gow ${ }^{29}$, Ruth B. Murray ${ }^{10}$, Nikolaos G. Papadopoulos ${ }^{30,31}$, Luis Perez-de-Llano ${ }^{32}$, Matthew Peters ${ }^{33}$, Paul E. Pfeffer ${ }^{34}$, Todor A. Popov ${ }^{35}$, Celeste M. Porsbjerg ${ }^{36}$, Chris A. Price ${ }^{10}$, Chin K. Rhee ${ }^{37}$, Mohsen Sadatsafavi $^{38}$, Yuji Tohda ${ }^{22}$, Eileen Wang ${ }^{39}$, Michael E. Wechsler ${ }^{40}$, James Zangrilli ${ }^{2}$ and David B. Price ${ }^{10,41,42^{*}}$

\begin{abstract}
Background: Severe asthma exerts a disproportionately heavy burden on patients and health care. Due to the heterogeneity of the severe asthma population, many patients need to be evaluated to understand the clinical features and outcomes of severe asthma in order to facilitate personalised and targeted care. The International Severe Asthma Registry (ISAR) is a multi-country registry project initiated to aid in this endeavour.

Methods: ISAR is a multi-disciplinary initiative benefitting from the combined experience of the ISAR Steering Committee (ISC; comprising 47 clinicians and researchers across 29 countries, who have a special interest and/or experience in severe asthma management or establishment and maintenance of severe asthma registries) in collaboration with scientists and experts in database management and communication. Patients ( $\geq 18$ years old) receiving treatment according to the 2018 definitions of the Global Initiative for Asthma (GINA) Step 5 or uncontrolled on GINA Step 4 treatment will be included. Data will be collected on a core set of 95 variables identified using the Delphi method. Participating registries will agree to provide access to and share standardised anonymous patient-level data with ISAR. ISAR is a registered data source on the European Network of Centres for Pharmacoepidemiology and Pharmacovigilance. ISAR's collaborators include Optimum Patient Care, the Respiratory Effectiveness Group (REG) and AstraZeneca. ISAR is overseen by the ISC, REG, the Anonymised Data Ethics \& Protocol Transparency Committee and the ISAR operational committee, ensuring the conduct of ethical, clinically relevant research that brings value to all key stakeholders.

(Continued on next page)
\end{abstract}

\footnotetext{
* Correspondence: dprice@opri.sg

${ }^{10}$ Optimum Patient Care, Cambridge, UK

${ }^{41}$ Observational and Pragmatic Research Institute, Singapore, Singapore

Full list of author information is available at the end of the article
}

(c) The Author(s). 2020 Open Access This article is licensed under a Creative Commons Attribution 4.0 International License, which permits use, sharing, adaptation, distribution and reproduction in any medium or format, as long as you give appropriate credit to the original author(s) and the source, provide a link to the Creative Commons licence, and indicate if changes were made. The images or other third party material in this article are included in the article's Creative Commons licence, unless indicated otherwise in a credit line to the material. If material is not included in the article's Creative Commons licence and your intended use is not permitted by statutory regulation or exceeds the permitted use, you will need to obtain permission directly from the copyright holder. To view a copy of this licence, visit http://creativecommons.org/licenses/by/4.0/ The Creative Commons Public Domain Dedication waiver (http://creativecommons.org/publicdomain/zero/1.0/) applies to the data made available in this article, unless otherwise stated in a credit line to the data. 
(Continued from previous page)

Conclusions: ISAR aims to offer a rich source of real-life data for scientific research to understand and improve disease burden, treatment patterns and patient outcomes in severe asthma. Furthermore, the registry will provide an international platform for research collaboration in respiratory medicine, with the overarching aim of improving primary and secondary care of adults with severe asthma globally.

Keywords: Disease registry, Protocol, Real-world, Severe asthma

\section{Background}

Treatment of severe asthma is challenging because of the heterogeneous nature of the disease, which comprises various phenotypes and endotypes [1-3]. The heterogeneity of airway inflammation in patients with severe asthma makes profiling of patients considerably important toward identifying "treatable traits" [4]. Moreover, while severe asthma affects approximately $5 \%-15 \%$ of all patients with asthma [5-7], the associated impact on the lives of patients and caregivers is disproportionately high $[8,9]$. Inadequately controlled severe asthma is also associated with poor clinical outcomes and a high economic burden [9-12].

National and local asthma registries are a source of realworld data for asthma management and may contain data at the patient, provider and clinic levels [13-17]. However, because of considerable differences in the definitions of severe asthma [5, 18-20] and country- and region-specific differences in access to health care [21, 22], patient populations included in these registries may be heterogeneous in terms of demographics, clinical characteristics, inflammatory phenotypes, captured data and reported outcomes. Additionally, depending on the size of existing asthma registries, they may be limited by inadequate statistical power. As a result of this heterogeneity, interpretation of data across patient populations and geographies can be challenging. Therefore, a standardised global dataset is urgently needed to:

- retain all the values of local registries and connect them to enable and promote inter-operability, data sharing and cross-comparison;

- have sufficient statistical power to answer pertinent clinical and research questions;

- reduce the variability of data collected by standardising variables across countries and regions;

- have pre-defined and extensive processes in place to ensure that data capture and data harmonisation are of high quality;

- improve understanding of the severe asthma population and examine the response to therapies and other interventions as a function of nationality, phenotypes, biomarkers, current treatment and socio-economic status; and

- permit continued development with long-term patient follow-up to enable a real-life understanding of severe asthma.
The International Severe Asthma Registry (ISAR) is a collaborative initiative comprising existing and new registries that builds on data from multiple nations and regions and increases the statistical power and comparability of data. It is the first global adult severe asthma registry to be established. The primary objectives of ISAR (Fig. 1) are to describe and characterise the natural history of the severe asthma patient population overall and by different subgroups and to facilitate phenotyping and endotyping of patients with severe asthma such that these patient groups can be described by burden of illness, disease management patterns and clinical evolution in an international setting. These objectives were developed to improve understanding of the clinical features and outcomes of severe asthma, with the overarching aim of improving the care of adults with severe asthma globally.

Here, we describe the ISAR protocol for registry development and management, the rationale behind each step of the process and the potential benefits of ISAR to adult patients with severe asthma.

\section{Methods}

\section{Registry design and governance}

ISAR, a multi-country, observational initiative, will both prospectively and retrospectively collect data on adult patients with severe asthma. A registered data source on the European Network of Centres for Pharmacoepidemiology and Pharmacovigilance (ENCePP) [23], ISAR is currently supported by three core collaborators: Optimum Patient Care (OPC), the Respiratory Effectiveness Group (REG) and AstraZeneca. OPC is a not-forprofit social enterprise providing medical research and services to improve the diagnosis, treatment and care of chronic diseases [24] and is responsible for ISAR database management, data processing and analyses. REG is an investigator-led, not-for-profit research initiative promoting the value of real-life research [25]. AstraZeneca and OPC are co-founders and joint sponsors of ISAR.

\section{Oversight}

ISAR is overseen by four governing bodies (Fig. 2): the ISAR Steering Committee (ISC), REG, the Anonymised Data Ethics \& Protocol Transparency (ADEPT) Committee [26] and the ISAR Operational Committee. 


\section{Primary Objectives}

- Describe and characterise the severe asthma patient population natural history overall, where appropriate, and by different subgroups

- Facilitate phenotyping and endotyping of patients with severe asthma and describe these groups by burden of illness, disease management patterns and clinical evolution in an international setting

\section{Secondary Objectives}

- Evaluate real-life effectiveness and safety of treatments for severe asthma overall and in specific patient groups/phenotypes

- Support development of effective and efficient diagnostic routines and therapeutic principles

- Improve patient outcomes through structured asthma reviews (short-term) and increased understanding of severe asthma (long-term)

- Minimise side effects of steroid exposure through use of appropriate treatments

- Assess differences in levels of classification as defined by comparing physician-diagnosed severe asthma at baseline against diagnostic criteria of established guidelines

- Describe disease management patterns such as treatment changes over time (e.g. step up, step down and switches), as well as reasons for changes, and the effect of these changes on clinical progression

- Describe factors associated with treatment choice at baseline

- Describe risk factors associated with poor asthma outcomes including exacerbations, disease burden, health care resource use, medication side effects and disease progression

- Assess the occurrence of exacerbations and other conditions, such as upper respiratory tract infections, including seasonal variations

- Assess biomarker data and estimate their predictive value for disease diagnosis phenotype/endotype characterisation, response to treatment and progression

- Identify patients who may be eligible for participation in future research studies

Fig. 1 Objectives of the International Severe Asthma Registry

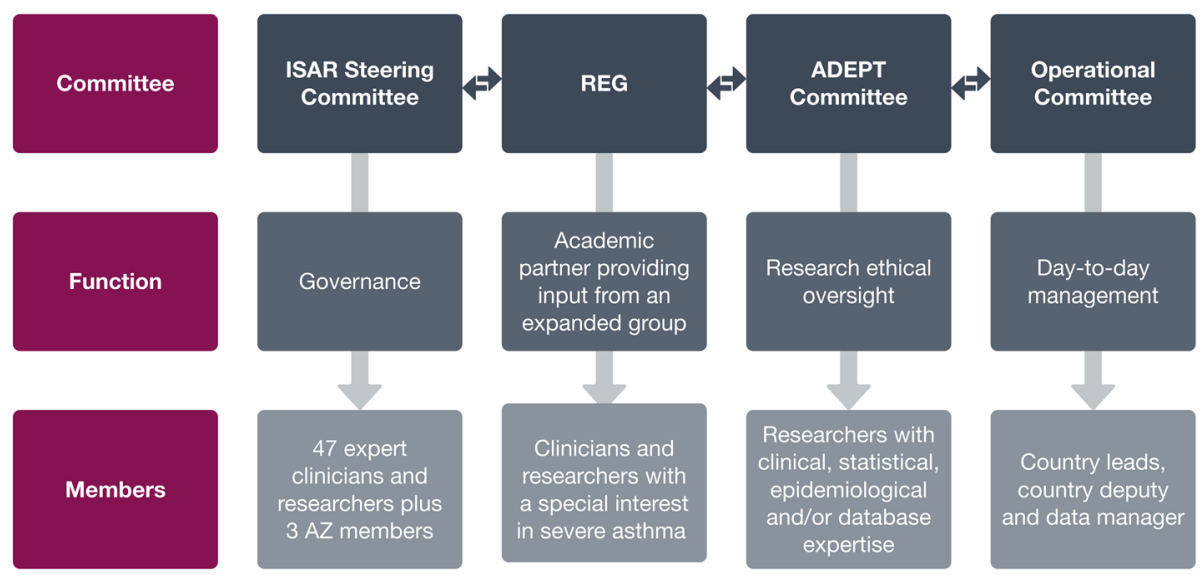

Fig. 2 International Severe Asthma Registry governance. ADEPT Anonymised Data Ethics \& Protocol Transparency, AZ AstraZeneca, ISAR International Severe Asthma Registry, REG Respiratory Effectiveness Group 
The ISC comprises 47 clinicians and researchers across 29 countries in North America, South America, Europe, Asia, Middle East and Oceania, with a special interest in severe asthma and/or experience in establishing and maintaining a severe asthma registry. The ISC also includes members of OPC and medical experts from AstraZeneca. Since ISAR is an open registry, academic and commercial entities can register their interest to participate in ISAR and seek access to the data for research purposes, with all research proposals requiring approval by the ISC via a democratic voting process. Each member country and AstraZeneca have one vote on project selection, with OPC holding the casting vote in the event of ties. AstraZeneca does not vote on research proposals from commercial entities.

REG is the academic partner comprising over 420 experts in severe asthma and will provide academic oversight and support. The ADEPT Committee (commissioned independently by REG), using prespecified criteria [24], will review and approve the scientific merit of all research proposals submitted to ensure scientific integrity, robustness and compliance with all relevant ethical considerations and to ensure that the database research is clinically appropriate and valuable to patients, public health and health care.

The ISAR Operational Committee includes participating country representatives (e.g. country lead, deputy and data managers) and will be involved in the day-today running of ISAR. The Operational Committee within each country is led by the country-specific ISC member; therefore, sound procurement of data is also partly ensured by the ISC.

\section{Registries, countries and experts}

Existing registries were selected for possible collaboration via a systematic internet search of PubMed, MEDLINE, EMBASE, Google Scholar and Web of Science using broad search terms to identify all severe asthma registries. Registries for participation in ISAR were identified with the help of REG's and OPC's global network of leading respiratory scientists and severe asthma experts. These experts were either the lead of an existing registry, were linked with the lead or had previously expressed an interest in developing a severe asthma registry for their respective country. Leaders of existing and potential new severe asthma registries were approached and formally invited by ISAR to discuss collaboration and contribution to the ISAR initiative. Table 1 lists the existing and new registries collaborating with ISAR.

To facilitate global collaboration efforts, various aspects of the ISAR structure and deliverables, such as principles of data collection and sharing, as well as research prioritisation, will be jointly discussed between
OPC and the lead entity for each registry/country and will be tailored towards each contributing country during the ISC meetings as well as country-specific meetings. The lead entity, depending on the local circumstances, could either be a university, a lead study site or the respective national thoracic society. In all instances, the lead entity is responsible for overseeing data collection, including combining data from any satellite sites, before making the country-wide data available to ISAR. In countries with no existing registries, this approach, in effect, creates a country-level registry that allows for the creation of a locally hosted central registry for the country's combined data, which can be used to enhance local-level research, and provides longitudinal data that will help clinicians and patients better understand underlying phenotypes of severe asthma and enhance precision medicine.

The ethics application process differs among collaborating registries. Within some registries, ethics approval is required at each individual satellite site, whereas for other collaborators, a central ethics committee can process applications on behalf of all satellite sites.

In addition, the ISAR study group comprises core panel members of ISAR who will provide collective expertise, scientific knowledge and experience in database management and research (Additional file 1: Appendix).

\section{Patients}

Patients in ISAR will be included from the participating existing and newly created local/regional registries. To be included, patients ( $\geq 18$ years of age) should be receiving treatment according to the 2018 definitions of the Global Initiative for Asthma (GINA) Step 5 or should be uncontrolled on GINA Step 4 treatment [19, 27]. Uncontrolled asthma is defined as per the American Thoracic Society/European Respiratory Society criteria [5]. Detailed inclusion and exclusion criteria are listed in Table 2. These eligibility criteria were chosen to reflect severe asthma patients in the real-world setting and to broaden the scope to include patients with uncontrolled moderate-to-severe asthma. Notably, patients with a history of smoking are not excluded. In addition, patients with asthma-chronic obstructive pulmonary disease overlap (ACO), as defined in the 2018 GINA report [19], will be included. Informed consent will be obtained from patients where required to allow anonymised data sharing for approved research projects.

On average, 2000 new patients will be enrolled globally each year, for at least 5 years from the start of ISAR (May 2017). Patients receiving care at severe asthma secondary and tertiary care centres in each participating country will be included, in accordance with local regulatory/ethics requirements. One follow-up per year is required with collaborating centres, which is in line with 
Table 1 List of existing and new registries collaborating with ISAR

\begin{tabular}{|c|c|c|c|}
\hline Registry status & Collaborating country & Registry name & Start year \\
\hline \multirow[t]{8}{*}{ Existing Registry } & UK & UK Severe Asthma Registry & 2006 \\
\hline & USA & National Jewish Health Electronic Medical Record (NJH EMR) & 2010 \\
\hline & South Korea & $\begin{array}{l}\text { Severe Asthma Work Group of Korean Academy of Asthma, } \\
\text { Allergy and Clinical Immunology (KAAACI) }\end{array}$ & 2010 \\
\hline & Germany & German Asthma Network (GAN) & 2011 \\
\hline & Australia \& New Zealand & Australasian Severe Asthma Registry (ASAR) hosted by TSANZ & 2013 \\
\hline & Ireland & INhaler Compliance Assessment in Severe Unstable Asthma (INCA SUN) & 2015 \\
\hline & Italy & Severe Asthma Network Italy (SANI) & 2016 \\
\hline & Spain & Spanish Guideline on the Management of Asthma Database (GEMA-Data) & 2017 \\
\hline \multirow[t]{25}{*}{ New Registry } & Denmark & Danish Severe Asthma Registry (DSAR) & 2018 \\
\hline & Sweden & Swedish Severe Asthma Registry & Starting in 2020 \\
\hline & Finland & Currently collecting data independently from ISAR & 2019 \\
\hline & Iceland & Currently collecting data independently from ISAR & 2020 \\
\hline & Norway & & Starting in 2021 \\
\hline & Bulgaria & Bulgarian Severe Asthma Registry (BULSAR) & 2018 \\
\hline & Portugal & Portugal Severe Asthma Registry (Registo de Asma Grave Portugal [RAG]) & 2018 \\
\hline & Russia & Russian Severe Asthma Registry (RSAR) & 2018 \\
\hline & Argentina & Argentinian Severe Asthma Registry & 2019 \\
\hline & Belgium & Currently collecting data independently from ISAR & 2018 \\
\hline & Brazil & Brazilian Severe Asthma Registry & Starting in 2020 \\
\hline & Canada & Canadian Severe Asthma Registry & 2019 \\
\hline & China & & Starting in 2021 \\
\hline & Colombia & Colombian Severe Asthma Registry & 2019 \\
\hline & France & French Severe Asthma Registry & 2019 \\
\hline & Greece & Greek Severe Asthma Registry & 2019 \\
\hline & India & Indian Severe Asthma Registry & 2019 \\
\hline & Japan & Japanese Severe Asthma Registry & 2019 \\
\hline & Kuwait & Kuwaitian Severe Asthma Registry & 2018 \\
\hline & Mexico & Mexican Severe Asthma Registry & 2019 \\
\hline & Poland & Polish Severe Asthma Registry & 2020 \\
\hline & Saudi Arabia & Saudi Arabian Severe Asthma Registry & 2019 \\
\hline & Singapore & Singapore Severe Asthma Registry (S-SAR) & 2020 \\
\hline & Taiwan & Taiwanese Severe Asthma Registry & 2019 \\
\hline & UAE & UAE Severe Asthma Registry & 2019 \\
\hline
\end{tabular}

ISAR International Severe Asthma Registry, UAE United Arab Emirates, UK United Kingdom, USA United States of America

the minimum number of visits to a severe asthma specialist centre expected per patient. A minimum of twothirds of patients enrolled within each participating registry are predicted to be retained for annual followup, after adjusting for attrition.

\section{Database}

The ISAR initiative is a partnership with national and regional registries, such that each participating registry retains ownership of their own data, but shares their anonymised and de-identified data with ISAR for approved research purposes.

A collaboration and data sharing agreement will be negotiated and signed between OPC and the lead entity of each registry. The agreement will govern the:

- method of storage and transmission of data;

- data security and compliance with OPC data security standards;

- list of variables required to be extracted from each country-specific database; 
Table 2 ISAR patient inclusion and exclusion criteria

\begin{tabular}{ll}
\hline Inclusion & Exclusion \\
\hline Adult ( $\geq 18$ years old) patients with severe asthma & Lack of informed consent for participation
\end{tabular}

Undergoing GINA Step 5 treatment ${ }^{\mathrm{a}}$ [19] or

Uncontrolled on GINA Step 4 treatment [19]

Uncontrolled defined as at least one of the following (per ATS/ERS guidelines [5]):

Poor symptom control: ACQ consistently $>1.5$, ACT $<20$ (or 'not well controlled') [19]

Airflow limitation: Pre-bronchodilator $\mathrm{FEV}_{1}<80 \%$ predicted, with reduced $\mathrm{FEV}_{1} / \mathrm{FVC}$

(defined as less than the lower limit of normal)

Serious exacerbations: $\geq 1$ hospitalisation, ICU stay or mechanical ventilation in the previous year

Frequent severe exacerbations: $\geq 2$ bursts of systemic corticosteroids with each course $>3$ days in the previous year

${ }^{a}$ Asthma controlled on high-dose ICS/LABA treatment was not part of the current inclusion for ISAR as this treatment approach is not yet adopted by clinicians ACQ Asthma Control Questionnaire, ACT Asthma Control Test, ATS American Thoracic Society, ERS European Respiratory Society, FEV 1 forced expiratory volume in $1 \mathrm{~s}$, FVC forced vital capacity, GINA Global Initiative for Asthma, ICS inhaled corticosteroids; ICU intensive care unit, ISAR International Severe Asthma Registry, LABA long-acting $\beta_{2}$-agonist

- oversight (by OPC) to ensure confidentiality of the data received; and

- remote retrieval and appraisal of data from each country by OPC.

The steps involved in data acquisition, quality control and management of ISAR have been outlined in Fig. 3.

\section{Data collection}

Data will be collected from a combination of existing and new registries, and each country will have a data collector trained in human subject research with relevant experience in Good Clinical Practice or country-specific equivalent guidelines. A small and limited financial incentive is also provided to compensate for any time

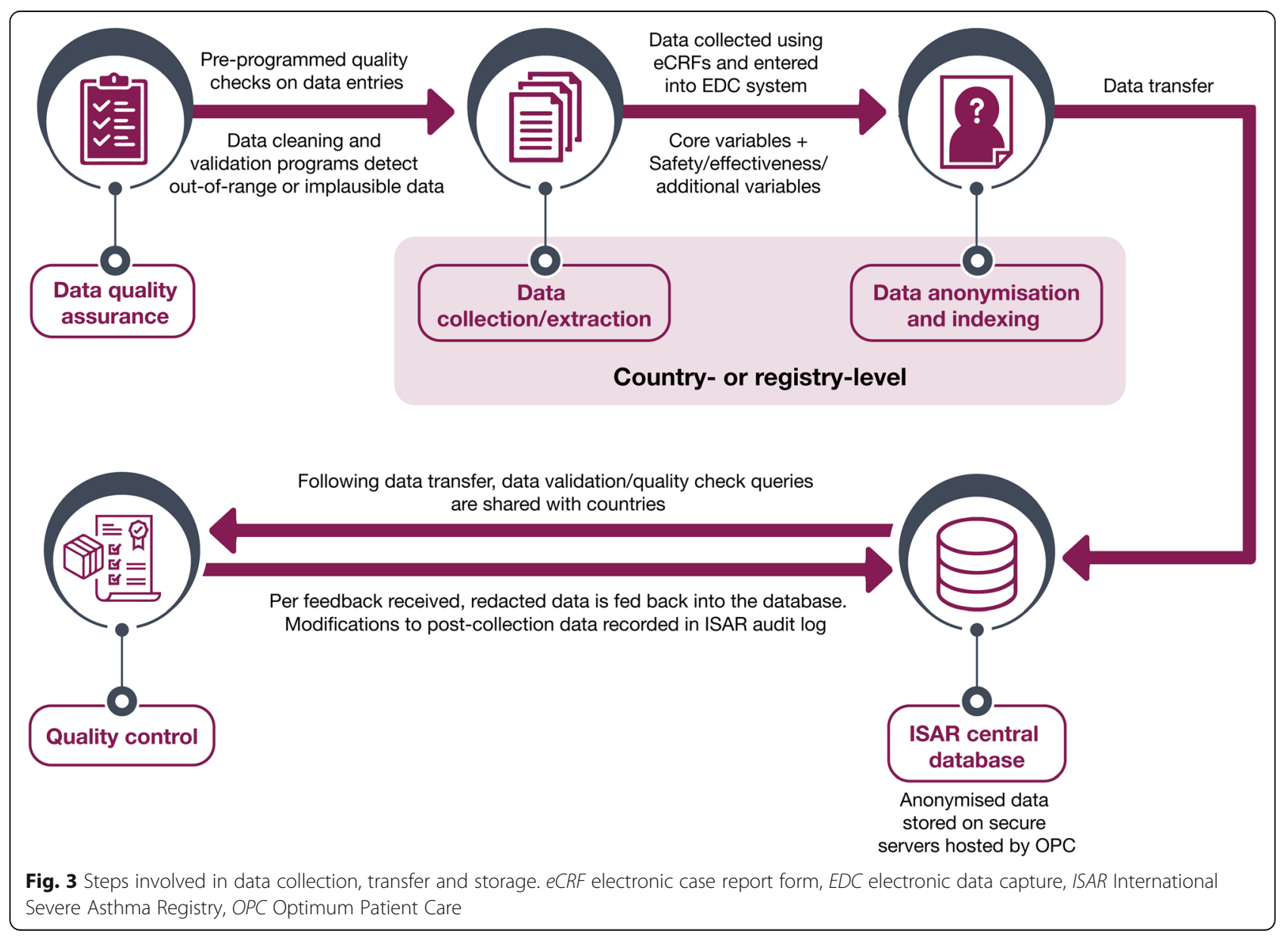


spent on data entry by health care providers or allied workers. To accommodate the recently enacted European Union General Data Protection Regulation (EU GDPR) [28], and/or local ethics regulatory mandates, some countries will provide summarised statistics for research projects, while others will provide individual-level data in the initial stage of the ISAR collaboration. However, all registries (as required) plan and work with ethics committees to transfer individual-level data. ISAR will collect only anonymised data from all collaborating registries and ensure that the strictest security measures are in place for data sharing and hosting. A data transfer standard operating procedure (SOP) is provided to each registry to guide the anonymous and safe transfer of data.

Data from the existing registries will be collected using existing systems (e.g. Dendrite Clinical Systems, United Kingdom [UK]; REDCap, Australia; Zitelab, Denmark) that are largely aligned with the standard data collection fields of ISAR. Data imported will be as per instructions listed in a separate ISAR data management plan, which will be provided to all registries. Data will be collected using a comprehensive electronic case report form (eCRF; screenshot in Fig. 4). Although registries can enter data directly in the eCRFs, they can also opt to collect the data on paper and enter it into the eCRF later, based on their clinical process. All eCRFs will be completed by designated personnel trained (via an on-site/ remote training session) on data entry, and a data collection SOP will be provided with instructions on how to complete the CRF/eCRF, with a detailed explanation of the data fields. Data collection will comply with the standards established by the ISC and agreed by each participating registry, allowing datasets across all registries to be combined. These procedures are set in place to ensure uniform interpretation of variables across countries and cultures, further effectively standardising ISAR data.

\section{Data quality and management}

Functions, processes and specifications for data collection, extraction, delivery and cleaning are outlined in the ISAR data management plan. For instance, data quality is ensured before and during the data collection process through a series of pre-programmed data quality checks

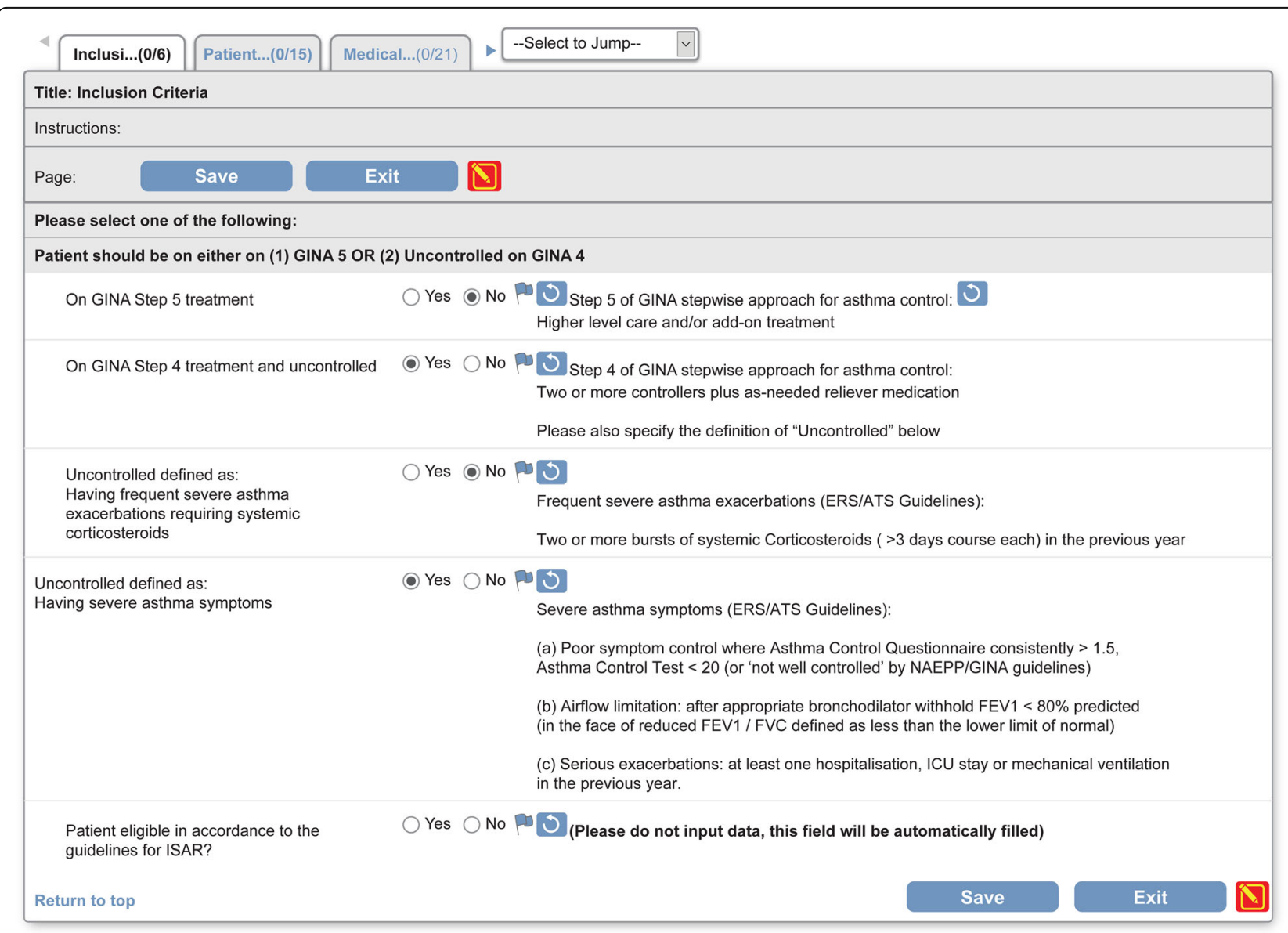

Fig. 4 International Severe Asthma Registry electronic case report form (screenshot). ATS American Thoracic Society, ERS European Respiratory Society, FEV1 forced expiratory volume in 1 second, FVC forced vital capacity, NAEPP National Asthma Education and Prevention Program 
that automatically detect out-of-range or anomalous entries on the eCRF. Most of the fields requested on the ISAR eCRF are numeric or categorical to minimise data entry errors. After data extraction, further data cleaning and validation processes will also be performed on all data to maximise data quality control. Ad hoc queries, done at the country level or OPC level, will be generated within the electronic data capture (EDC) system and followed up with country data managers and/or the country study coordinator (where applicable) for resolution. All data modifications will be recorded in an audit $\log$ and all data transfers and disputes will be shared and documented in the country and ISAR central data manager logs.

\section{Electronic data capture}

All new data will be entered directly into the EDC system (REDCap or OpenClinica). Where feasible, patient data from electronic medical records will be integrated with the EDC systems to maximise data collection resources and reduce the time needed for data entry. Both new and existing data collection platforms will accommodate ISAR variables and anonymise and de-identify data prior to importing it to the central data warehouse, where the data will be stored with a unique patient identification number. The key code linking the unique identifier to the relevant patient will be held by the patient's health care provider. ISAR will have no access to the linkage files. All participating sites will have access to their own data and will be trained on using the available online data capture systems. Physician and data entry personnel will be able to access their local EDC account with a username and password, with each user being prompted to change their unique password on a yearly basis.

OPC will be responsible for monitoring and mapping the data into the central ISAR data repository by using the Systematized Nomenclature of Medicine Clinical Terms (SNOMED CT) code [29] for standardised use globally. In addition, all existing registries using unique EDC systems will be responsible for extracting batches of patient data at a quarterly frequency for inclusion to ISAR. OPC will be responsible for safely transporting and importing each batch into the central ISAR data repository permitted by specific country's data regulations.

For countries with data sharing regulations delimiting data privacy, such as the EU GDPR, ISAR will accommodate anonymised data sharing on a project-by-project basis. In these cases, the patient-level data are kept/ hosted within the country and shared as needed for approved ISAR research.

\section{Registry variables}

A modified Delphi consensus-driven approach [30] was used to develop a standardised set of core and research variables for ISAR [31]. The ISC will hold regular meetings to ensure continued expert input throughout the development and expansion of the registry, in terms of the collaborators and investigators added, as well as data collected. Standardised data will be collected in three distinct fields:

Core variables These comprise 13 categories encompassing 95 core variables (Fig. 5; Additional file 2: Table S1), which include data on patient demographics, medical history and diagnostics, clinical characteristics, patient-reported outcomes and treatment management plans. These core variables are mandatory and should be collected by any registry wishing to contribute data to ISAR. Additional variables (e.g. those collected by the UK registry) can be reviewed by each ISAR member and included into the core variable list. However, no change is planned in the core variables for the first 5 years of ISAR. Each country is permitted to keep any number of

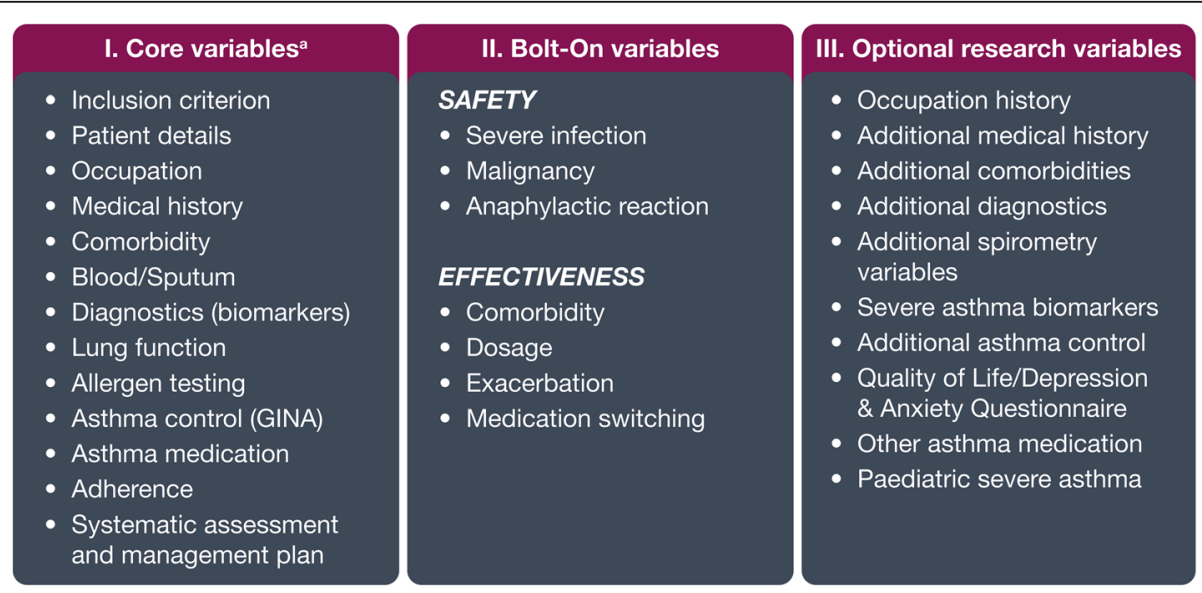

Fig. 5 International Severe Asthma Registry variables. ${ }^{a}$ Collected by all participating registries. GINA Global Initiative for Asthma 
these additional variables as extended variables in their own local registry/database.

Safety and effectiveness bolt-on variables Safety variables (Fig. 5; Additional file 3: Table S2) comprising severe infections, malignancy and anaphylactic reactions were chosen because of evidence indicating the potential association between the use of biologics and these adverse events [32, 33]. Considering that these outcomes and associations have mostly been studied in small populations, the inclusion of these safety bolt-on variables will enable investigation and evaluation of these in a larger, global population with severe asthma.

Similarly, the effectiveness variables (Fig. 5; Additional file 3: Table S2) were selected to collect indications of osteoporosis, circulatory system diseases, ocular diseases (glaucoma and cataract), renal failure, type II diabetes mellitus, depression, anxiety, pneumonia, peptic ulcer, obstructive sleep apnoea and details on oral and inhaled corticosteroid doses, as well as reasons for medication therapy switches.

Additional research variables These comprise all variables deemed useful for scientific research and maximal data collection, such as occupation, medical history, additional comorbidities, diagnostics, spirometry variables, biomarkers, asthma control, quality of life or depression and anxiety questionnaire, other asthma medications and paediatric severe asthma (Fig. 5; Additional file 4: Table S3). Extended variables will be collected via standardised bolt-on modules and will be available by choice to registries according to their preference. If resources allow, each registry is free to add additional variables of specific local interest, such as pregnancy, to their data collection tool.

\section{Data ownership}

Each country retains ownership of their data, and all participating countries agree to allow output of data from their respective registries upon joining ISAR for collaborative independent research approved by the ISC and ADEPT. The extraction and integration of datasets for ethically approved research studies will be managed by $\mathrm{OPC}$, and the nature and frequency of data extraction and transfer (quarterly) from registries to OPC are detailed in the ISAR data sharing agreement.

\section{Research}

In terms of research output, the interim research goal of ISAR is to complete one global research project per year and to create four additional project-specific datasets for academic and commercial research by ISAR members. Non-core research activities will be presented as an additional two abstracts and two manuscripts per year from 2018 to 2021.

While ISAR will continue to actively seek out new partners, new collaborators may also join ISAR by using the 'Join Us or Register Interest' option on the ISAR home page (http://isaregistries.org/). Research ideas may be suggested by ISC members, country leads, and contributors and visitors to the ISAR website (which may include third party commercial and academic research organisations), by clicking the 'Submit Proposal or Request Research' tab on the ISAR home page. All research ideas will be reviewed, assessed and prioritised by the ISC. Notably, regardless of commercial partners or collaborators, there will be no inferential drug-to-drug comparison in ISAR; however, in the context of baseline patient characteristics and treatment patterns (e.g. switching), the proportions of patients on specific biologics may be described and comparisons of outcomes may be made between different biologics by class.

ISAR is open to other datasets that are not part of the core ISAR projects but have alignment of variables, which will enable combining data for specific projects. Thus, combining and collaborating with other databases or extracting data from other databases for specific projects is an available option.

\section{Discussion}

ISAR is a global collaborative initiative that allows for prospectively and retrospectively analysing real-life severe asthma data at the patient-level. It is conducted by $\mathrm{OPC}$, with academic and regulatory oversight from the ISC, academic support from REG, ethical governance from ADEPT and joint funding support from OPC and AstraZeneca. ISAR is facilitated by standard data collection via a core set of variables across all participating registries and supporting data collection via electronic data capture, hosting and data entry. Benefitting from the use of aggregate vs. individualised data, ISAR is large enough to provide sufficient statistical power to detect differences and trends. It thus enables collaborating registries to answer key research questions on asthma at a global scale.

Currently, ISAR's membership includes registries from more than 30 participating countries (Fig. 6). The inclusive nature of ISAR will allow for extensive collaboration and potentially new research ideas. To ensure transparency, ISAR has in place a democratic voting system for selection of core research projects. In addition, data quality checks run by ISAR may lead to a robust dataset enabling quality research if countries decide to conduct their own research with their local data. Overall, ISAR aims to consolidate current knowledge of severe asthma based on six key strengths (Fig. 7), including its global reach, high quality data drawn from a large sample size, 


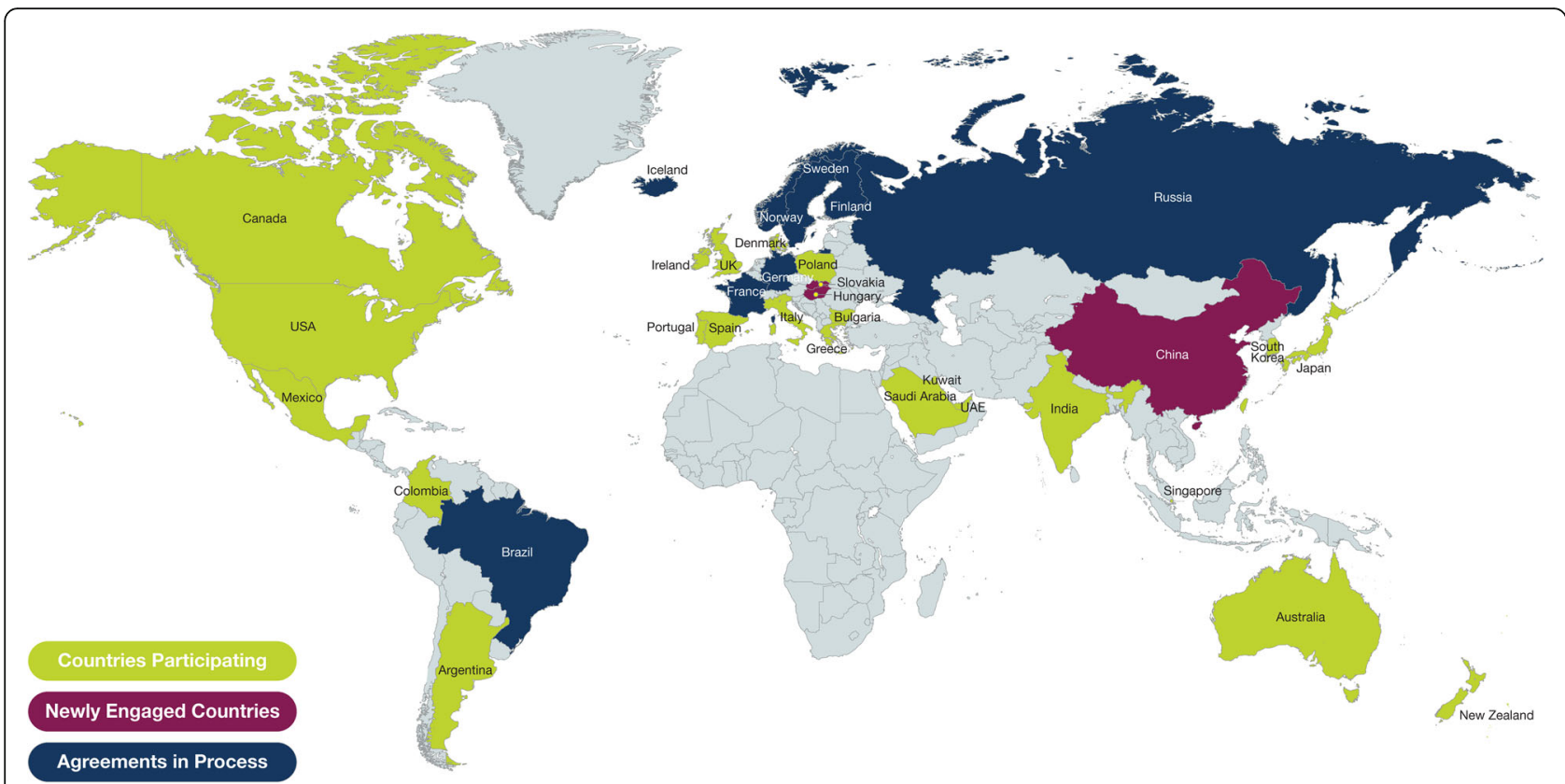

Fig. 6 Current International Severe Asthma Registry snapshot ${ }^{a}$. ${ }^{2}$ Source: image created in-house. UAE United Arab Emirates, UK United Kingdom, USA United States of America

organisational structure, experience with database management inclusivity and expertise, enabling the generation of research that can have an impact on patient care globally.

Certain limitations of ISAR should be acknowledged. First, any database is only as good as the data it contains (i.e. what is measured, in whom, how and the extent of missing data). Second, due to their design, registry data may possess lower internal validity than data collected prospectively in randomised clinical trials, limiting the extent to which they can support causal relationships. The large volume of data also creates the potential for many analyses with selective reporting, although this will be mitigated by open disclosure of the research conducted on the dataset and the respective results. While ISAR has a broad representation from a number of countries, for some, there may be limited representation within the country itself. For example, ISAR currently covers only one site in the United States. Lastly, to become a truly global representation of severe asthma,

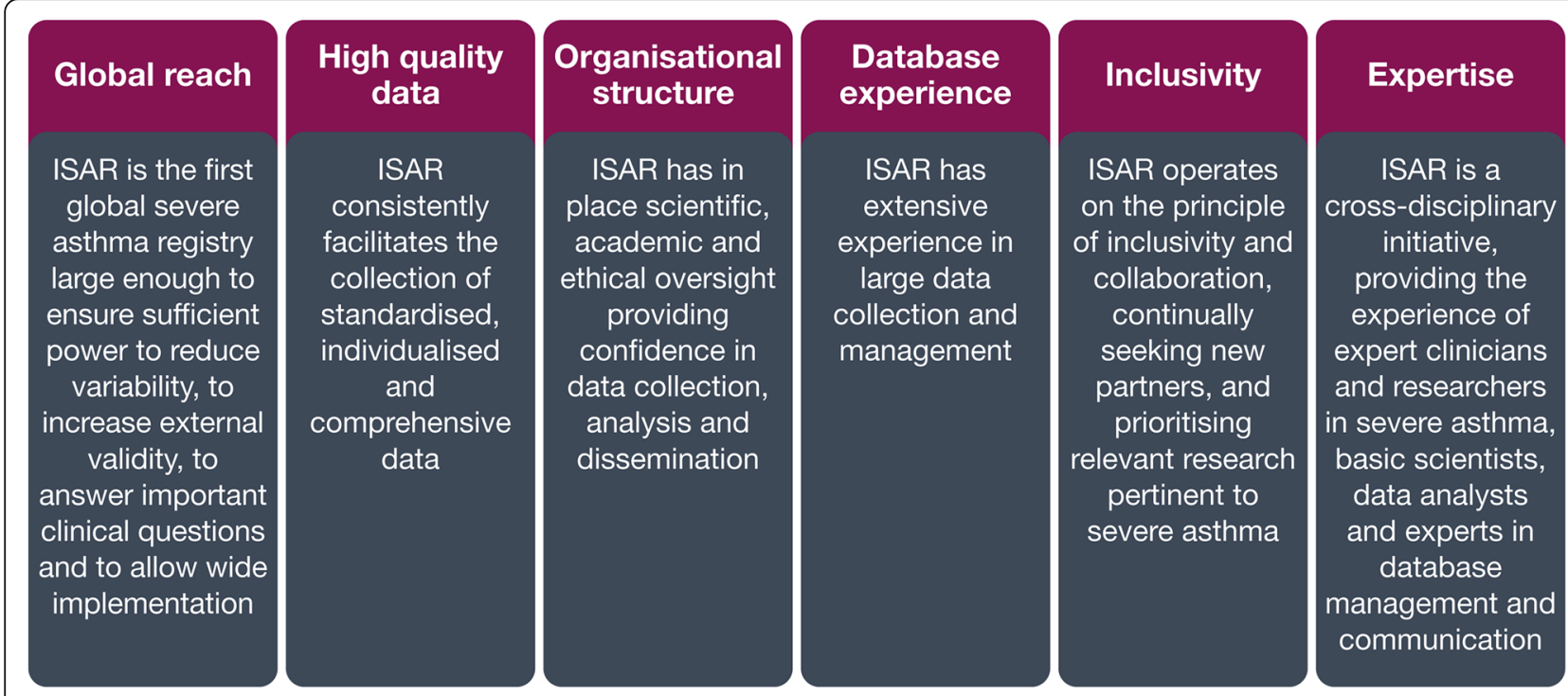

Fig. 7 Key strengths of the International Severe Asthma Registry. ISAR International Severe Asthma Registry 
funding issues need to be overcome to include patients from sub-Saharan Africa and other low-to-middle income countries.

The ISAR initiative has already led to the generation of key data in the severe asthma field. The research topic prioritised by the ISC in the inaugural year 2017 was the 'Demographic and clinical characteristics of severe asthma patients worldwide'. Characteristics described included demographic distribution, medication, comorbidities, asthma control, blood eosinophil counts, immunoglobulin $\mathrm{E}$ (IgE) levels, lung function and health care resource use. In total, 4990 patients from five registries contributed to this first research project of ISAR [34]. In 2018, the global research project was on the characterisation and comparison of eosinophilic and non-eosinophilic phenotypes (Manuscript under journal review). The primary objective was to describe and compare the demographic and clinical features of eosinophilic compared with non-eosinophilic asthma phenotypes in an international cohort of adult patients with severe asthma. The broader definitions of type 2 vs. non-type 2 asthma [35] will also be examined. The core project prioritised by the ISC for 2019 is the 'Comparative effectiveness across severe asthma biologic classes (anti-interleukin-5 vs. anti-IgE targeted therapy) in patients eligible for both modalities'. Furthermore, additional ISAR research projects are prioritised and are open to researchers to join and lead (Table 3). Overall, the clinical questions that ISAR plans to answer are varied and range from a description of the patient population to the assessment of any differences between disease subtypes, drivers of treatment switching, biomarker profiles and the development of a protocol to identify hidden severe asthma.

There is precedence for the use of global registries to facilitate international collaboration and contribute to our understanding of other diseases [36, 37]. For example, the Translational Research in Europe - Assessment \& Treatment of Neuromuscular Diseases (TREAT-NMD), which is a network for the neuromuscular field, enabled harmonised implementation of registries featuring patients with Duchenne's muscular dystrophy [36]. The European Register for Multiple Sclerosis (EUReMS) was created to enable comparisons across EU countries by merging data from existing national multiple sclerosis registries and regional cohorts [37]. In rheumatology, collaboration across global registries made access to rich data sources possible, enabling investigation of safety outcomes among a geographically diverse rheumatoid arthritis patient population [38]. Thus, ISAR has the potential to become an important platform to facilitate severe asthma research. Furthermore, the protocol described here can potentially be used as a foundation in other diseases, where divergent national and regional registries preclude collaboration and inter-operability between registries.

In the future, ISAR plans to include additional countries, covering Africa, Asia, South America, the Middle East and Eastern Europe. Other prospects include linkages with other databases and integration with electronic

Table 3 Prioritised research projects for 2018, 2019 and 2020

\begin{tabular}{|c|c|}
\hline Project & Investigator \\
\hline \multicolumn{2}{|l|}{2018} \\
\hline $\begin{array}{l}\text { Biologics in severe asthma: utilisation patterns, causes for discontinuation and switching and } \\
\text { adverse outcomes }\end{array}$ & Professor Andrew Menzies-Gow (UK) \\
\hline Hidden severe asthma patients in primary care vs. ISAR & Professor David Price (Singapore) \\
\hline Relationship between socioeconomic status and asthma outcomes & Professor Liam Heaney (UK) \\
\hline Biomarker Relatability in the International Severe Asthma Registry (BRISAR) & Dr. Eve Denton and Dr. Mark Hew (Australia) \\
\hline Identification of predictors (i.e. biomarkers) of response to biologics & Dr. Eve Denton and Dr. Mark Hew (Australia) \\
\hline Hidden chronic asthma within the COPD/ACO population & Professor Chin Kook Rhee (South Korea) \\
\hline Age of onset of asthma in severe asthma patients & Dr. Enrico Heffler (Italy) \\
\hline
\end{tabular}

2019

Describe the OCS landscape: annual consumption, prevalence, outcomes and side effects of long-term OCS users

Criteria for choosing and switching between similar biological treatment options in patients with atopic and non-atopic severe eosinophilic asthma

2020

What is the impact of co-morbidity in severe asthma?

Define responders and non-responders to biologics and describe their characteristics overall and per biologic

Describe the clinical outcome before and after biologic treatment by biologic class, by individual biologic, and by subgroups of baseline characteristics

$A C O$ asthma-chronic obstructive pulmonary disease overlap, COPD chronic obstructive pulmonary disease, $F E V_{1}$ forced expiratory volume in 1 second, ISAR International Severe Asthma Registry, OCS oral corticosteroids, UK United Kingdom 
medical records. In addition, longitudinal research in patients with less severe asthma and the development of a paediatric ISAR in order to cover the entire severe asthma life cycle are also being considered.

\section{Conclusion}

ISAR is the first global registry for adult severe asthma that captures a large volume of standardised, international data on severe asthma. By acting as a data custodian of international patient data, ISAR works as an open border initiative, providing a platform to facilitate data sharing. The registry provides enough statistical power to address important research questions in severe asthma aimed at a wide range of topics, including knowledge of patient presentations, disease heterogeneity and the natural history of severe asthma; diagnosis and disease stratification; identification of predictors of treatment success and new treatment targets; demonstration of how treatments are used in real-life and how effective they are; long-term safety in different patient populations and comparison of differences between countries and care systems. Through ISAR, it is expected that the harmonised, standardised nature of data contained and the collaborative partnerships being made possible may reveal previously unthought of or hitherto neglected research avenues.

\section{Supplementary information}

Supplementary information accompanies this paper at https://doi.org/10. 1186/s12874-020-01065-0.

\section{Additional file 1: Appendix. International Severe Asthma Registry} study group.

Additional file 2: Table S1. Full list of ISAR 95 core variables. Additional file 3: Table S2. International Severe Asthma Registry bolton variables.

Additional file 4: Table S3. International Severe Asthma Registry optional additional research variables.

\section{Abbreviations}

ACO: asthma-chronic obstructive pulmonary disease overlap; ACQ: Asthma Control Questionnaire; ACT: Asthma Control Test; ADEPT: Anonymised Data Ethics \& Protocol Transparency; ATS: American Thoracic Society; eCRF: Electronic Case Report Form; EDC: Electronic Data Capture; ENCePP: European Network of Centres for Pharmacoepidemiology and Pharmacovigilance; ERS: European Respiratory Society; EU GDPR: European Union General Data Protection Regulation; EUReMS: European Register for

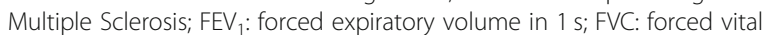
capacity; GINA: Global Initiative for Asthma; ICS: inhaled corticosteroids; ICU: intensive care unit; IgE: immunoglobulin E; ISAR: International Severe Asthma Registry; ISC: ISAR Steering Committee; LABA: Iong-acting $\beta_{2}$ agonist; OPC: Optimum Patient Care; REG: Respiratory Effectiveness Group; SNOMED $\mathrm{CT}$ : Systematized Nomenclature of Medicine Clinical Terms; SOP: standard operating procedure; TREAT-NMD: Translational Research in Europe Assessment \& Treatment of Neuromuscular Diseases; UK: United Kingdom

\section{Acknowledgements}

The authors thank the following individuals who contributed to the initial set-up of ISAR, provided input to the development of the protocol and/or were involved in local implementation of ISAR: Elisabeth Bel, Roland Buhl,
Sverre Lehmann, Stelios Loukidis, Richard Martin, Juno Pak, Pearlanne Zelarney, Joy Zimmer, Christena Kolakowski, Margo Brown, Jessica Cummings, Jennifer Brandorff, Seth Skelton, John Upham, Philip Bardin, Paul Reynolds, David Langton, Peter Middleton, Belinda Cochrane, Katya Vasileva Noleva, Plamen Hristov Yakovliev, Sonya Metodieva Genova, Violina Milchova Vasileva, Darina Petrova Dimova, Nadezhda K Takovska, Cvetantka Hristova Odzhakova, Eleonora M Stamenova, Diana X Hristova, Vincente Plaza, lan Hirsch, Cekomir Vodenicharov, Alexandrina Vodenicharova and Magdalena

Alexandrova.

Medical writing support was provided by Michelle Rebello, PhD, and Liam Gillies, PhD, of Cactus Communications (Mumbai, India).

\section{Authors' contributions}

AHM-vdZ, AA, AM-G, AB, BGC, CAP, CKR, CMP, DBP, DJJ, DL, EH, EW, GB, GC, GWC, JB, JMF, IC, JZ, LB1, LB2, LGH, LL, LP, MA, MEW, MH, MP, MS, MSK, NE, $\mathrm{NH}, \mathrm{NP}, \mathrm{PEP}, \mathrm{PG}, \mathrm{RBM}, \mathrm{RJ}, \mathrm{RWC}, \mathrm{TAP}, \mathrm{TI}, \mathrm{TL}, \mathrm{TNT}$, UB, VB, VC, YSC and YT made substantial contributions to conception and design of the protocol, took part in drafting the article or revising it critically for important intellectual content, gave final approval of the version to be published and agree to be accountable for all aspects of the work.

\section{Funding}

The International Severe Asthma Registry is conducted by Optimum Patient Care Global Limited, and co-funded by Optimum Patient Care Global Limited and AstraZeneca. ISAR is supported by grants from AstraZeneca and Optimum Patient Care (OPC) Global (a not-for-profit social enterprise). The ISAR steering committee (ISC) was involved in the development of the protocol and is responsible for approving research proposals via a democratic voting process. In addition to 47 clinicians and researchers with an interest and experience in severe asthma, the ISC also includes members of OPC and four medical experts from AstraZeneca. AstraZeneca reviewed the draft before submission; however, decision to submit was made by the authors. Medical writing support was funded by AstraZeneca in accordance with Good Publication Practice (GPP3) guidelines (http://www.ismpp.org/gpp3).

\section{Availability of data and materials} Not applicable.

\section{Ethics approval and consent to participate}

The ethics application process differs among participating registries/countries in ISAR. Within some registries, ethics approval is required at each individual satellite site, whereas for other collaborators, a central ethics committee can process applications on behalf of all satellite sites.

Informed consent will be obtained from patients where required to allow anonymised data sharing for approved research projects in ISAR.

\section{Consent for publication}

Not applicable.

\section{Competing interests}

AHM-vdZ declares unrestricted grants from GlaxoSmithKline, Novartis and Boehringer Ingelheim, personal fees for advisory board activities from AstraZeneca and a travel grant from Chiesi. AA has received lecture fees from AstraZeneca, Boehringer Ingelheim, Chiesi, GlaxoSmithKline, MSD, Norameda, Novartis and Orion; sponsorships from AstraZeneca, Boehringer Ingelheim, Chiesi, GlaxoSmithKline, MSD, Norameda and Novartis; and has been a member of advisory boards for AstraZeneca, Boehringer Ingelheim, GlaxoSmithKline, Novartis and Teva. AM-G declares grants from AstraZeneca, Boehringer Ingelheim, GlaxoSmithKline and Hoffman La Roche; consultancy agreements with AstraZeneca, Sanofi and Vectura; attendance at advisory boards for AstraZeneca, Boehringer Ingelheim, GlaxoSmithKline, Novartis, Sanofi and Teva; received speaker fees from AstraZeneca, Boehringer Ingelheim, Novartis, Teva and Vectura; and attended international conferences for Boehringer Ingelheim and Teva. AB has received speaker fees and grants to his institution from AstraZeneca, Boehringer Ingelheim, Chiesi, GlaxoSmithKline and Novartis for unrelated projects. CMP, DL, GC, JB, JMF, MS, RBM, TAP, $T L, U B, V B$ and YSC have no relevant conflicts of interest. CKR declares consultancy and lecture fees from AstraZeneca, Boehringer Ingelheim, GlaxoSmithKline, MSD, Mundipharma, Novartis, Sandoz, Takeda and Teva-Handok. CAP, IC, LB2, NE, NH and VC are employees of Optimum Patient Care, a cofunder of the International Severe Asthma Registry. DJJ has received advisory 
board and speaker fees from AstraZeneca, GlaxoSmithKline, Boehringer Ingelheim, Teva, Napp Pharmaceuticals, Chiesi and Novartis and research grant funding from AstraZeneca. DBP declares board membership with Aerocrine, Amgen, AstraZeneca, Boehringer Ingelheim, Chiesi, Mylan, Mundipharma, Napp Pharmaceuticals, Novartis and Teva; consultancy agreements with Almirall, Amgen, AstraZeneca, Boehringer Ingelheim, Chiesi, GlaxoSmithKline, Mylan, Mundipharma, Napp Pharmaceuticals, Novartis, Pfizer, Teva and Theravance; grants and unrestricted funding for investigator-initiated studies (conducted through Observational and Pragmatic Research Institute Pte Ltd) from Aerocrine, AKL Research and Development Ltd., AstraZeneca, Boehringer Ingelheim, British Lung Foundation, Chiesi, Mylan, Mundipharma, Napp Pharmaceuticals, Novartis, Pfizer, Respiratory Effectiveness Group, Teva, Theravance, UK National Health Service and Zentiva; payment for lectures/speaking engagements from Almirall, AstraZeneca, Boehringer Ingelheim, Chiesi, Cipla, GlaxoSmithKline, Kyorin, Mylan, Merck, Mundipharma, Novartis, Pfizer, Skyepharma and Teva; payment for manuscript preparation from Mundipharma and Teva; payment for the development of educational materials from Mundipharma and Novartis; payment for travel/accommodation/meeting expenses from Aerocrine, AstraZeneca, Boehringer Ingelheim, Mundipharma, Napp Pharmaceuticals, Novartis and Teva; funding for patient enrolment or completion of research from Chiesi, Novartis, Teva and Zentiva; stock/stock options from AKL Research and Development Ltd., which produces phytopharmaceuticals; owns $74 \%$ of the social enterprise Optimum Patient Care Ltd. (Australia and UK) and 74\% of Observational and Pragmatic Research Institute Pte Ltd. (Singapore); and is a peer reviewer for grant committees of the Efficacy and Mechanism Evaluation programme and Health Technology Assessment. EH has been on advisory boards for SanofiGenzyme, GlaxoSmithKline, Novartis, Circassia and Nestlè Purina. EW has received advisory board fees from AstraZeneca. She has been an investigator on clinical trials sponsored by AstraZeneca, GlaxoSmithKline, Novartis, Teva and National Institute of Allergy and Infectious Diseases (NIAID), for which her institution has received funding. BGC reports personal fees and nonfinancial support from Novartis, grants and personal fees from AstraZeneca, Chiesi and Menarini; personal fees and non-financial support from Teva, grants from Boehringer Ingelheim and personal fees from Sanofi and Mundipharma, outside the submitted work. GWC has received research grants as well as lecture or advisory board fees from Menarini, Alk-Abello, Allergy Therapeutics, Anallergo, AstraZeneca, Medlmmune, Boehringer Ingelheim, Chiesi Farmaceutici, Circassia, Danone, Faes, Genentech, Guidotti-Malesci, GlaxoSmithKline, Hal Allergy, Merck, MSD, Mundipharma, Novartis, Orion, SanofiAventis, Sanofi, Genzyme/Regeneron, Stallergenes, UCB Pharma, Uriach Pharma, Teva, Thermo Fisher and Valeas. GB has received honoraria for lectures from AstraZeneca, Boehringer Ingelheim, Chiesi, GlaxoSmithKline, Novartis and Teva. He is a member of advisory boards for AstraZeneca, Boehringer Ingelheim, GlaxoSmithKline, Novartis, Sanofi/Regeneron and Teva. JZ, MA and TNT are employees of AstraZeneca, a co-funder of the International Severe Asthma Registry. LL declares personal fees for consultancy and lectures from ALK, AstraZeneca, Boehringer Ingelheim, Chiesi, GlaxoSmithKline, Mundipharma, Novartis, Orion Pharma, Sanofi and Teva. LB1 has (in the last three years) received lecture or advisory board fees from Alk-Abello, AstraZeneca, Boehringer, Chiesi, GlaxoSmithKline, Mundipharma, Novartis, Sanofi, Genzyme/Regeneron and Teva. LGH declares he has received grant funding, participated in advisory boards and given lectures at meetings supported by Amgen, AstraZeneca, Boehringer Ingelheim, Circassia, Hoffmann la Roche, GlaxoSmithKline, Novartis and Teva; he has taken part in asthma clinical trials sponsored by Boehringer Ingelheim, Hoffmann la Roche and GlaxoSmithKline, for which his institution received remuneration; and he is the Academic Lead for the Medical Research Council Stratified Medicine UK Consortium in Severe Asthma, which involves industrial partnerships with a number of pharmaceutical companies, including Amgen, AstraZeneca, Boehringer Ingelheim, GlaxoSmithKline, Hoffmann la Roche and Janssen. LP declares nonfinancial support, personal fees and grants from Teva; non-financial support and personal fees from Boehringer Ingelheim, Esteve, GlaxoSmithKline, Mundipharma and Novartis; personal fees and grants from AstraZeneca and Chiesi; personal fees from Sanofi and non-financial support from Menarini, outside the submitted work. MH declares grants and other advisory board fees (made to his institutional employer) from AstraZeneca, GlaxoSmithKline, Novartis and Seqirus for unrelated projects. MP declares personal fees and non-financial support from AstraZeneca and GlaxoSmithKline. MEW reports receiving consulting honoraria from AstraZeneca, Boehringer Ingelheim, Genentech, GlaxoSmithKline, Novartis, Regeneron, Sanofi and Teva. MSK has been a member of advisory boards for AstraZeneca, GlaxoSmithKline and Sanofi and has received honoraria from AstraZeneca, GlaxoSmithKline and Sanofi. NP declares speaker fees and grants to his institution from AstraZeneca, GlaxoSmithKline and Novartis for unrelated projects. PEP declares speaker fees and grants to his institution from AstraZeneca, GlaxoSmithKline and Novartis for unrelated projects. PG declares speaker fees and grants to his institution from AstraZeneca, GlaxoSmithKline and Novartis for unrelated projects. RWC has received honoraria for lectures from Aerogen, AstraZeneca, Boehringer Ingelheim, GlaxoSmithKline, Novartis and Teva. He is a member of advisory boards for GlaxoSmithKline and Novartis, has received grant support from GlaxoSmithKline and Aerogen and has patents in the use of acoustics in the diagnosis of lung disease, assessment of adherence and prediction of exacerbations. RJ declares grants from Novartis, GlaxoSmithKline and AstraZeneca; personal fees for consultancy and speaker fees or travel support from GlaxoSmithKline, AstraZeneca, Boehringer Ingelheim, Chiesi, Nutricia, OPRI and Pfizer. Tl declares grants from Astellas, Boehringer Ingelheim, Daiichi-Sankyo, Kyorin, Meiji Seika Pharma and Teijin Pharma and lecture fees from Kyorin. YT declares honoraria from Kyorin Pharma and Teijin Pharma and research funding from Kyorin and Meiji Seika Pharma.

\section{Author details}

${ }^{1}$ The Institute for Heart Lung Health, Vancouver, Canada. ${ }^{2}$ AstraZeneca, Gaithersburg, USA. ${ }^{3}$ Department of Pulmonary Medicine, University of Tartu and Lung Clinic, Tartu University Hospital, Tartu, Estonia. ${ }^{4}$ Center of Physical Activity Research, Rigshospitalet and Copenhagen University, Copenhagen, Denmark. ${ }^{5}$ Department of Respiratory Medicine \& Allergology, Skåne University Hospital, Lund, Sweden. ${ }^{6}$ Faculty of Medicine, University of Iceland, Reykjavik, Iceland. ${ }^{7}$ Department of Respiratory Diseases, Montpellier University Hospitals, Hopital Arnaud de Villeneuve and PhyMed Exp (INSERM U 1046, CNRS UMR9214), Universite de Montpellier, Montpellier, France. ${ }^{8}$ Department of Respiratory Medicine, Ghent University Hospital, Ghent, Belgium. ${ }^{9}$ Departments of Epidemiology and Respiratory Medicine, Erasmus Medical Center Rotterdam, Rotterdam, The Netherlands. ${ }^{10}$ Optimum Patient Care, Cambridge, UK. ${ }^{11}$ Centre for Public Health, Queen's University Belfast, Belfast, UK. ${ }^{12}$ Personalized Medicine Asthma \& Allergy Clinic, Humanitas University \& Research Hospital, Milan, Italy. ${ }^{13}$ SANI-Severe Asthma Network Italy, Milan, Italy. ${ }^{14}$ Division of Allergy, Department of Medicine, Asan Medical Center, College of Medicine, University of Ulsan, Seoul, South Korea. ${ }^{15}$ Faculty of Public Health, Medical University - Sofia, Sofia, Bulgaria. ${ }^{16}$ Son Espases University Hospital-IdISBa-Ciberes, Mallorca, Spain. ${ }^{17}$ Clinical Research Centre, Smurfit Building Beaumont Hospital and Department of Respiratory Medicine, RCSI, Dublin, Ireland. ${ }^{18}$ Australasian Severe Asthma Network, Priority Research Centre for Healthy Lungs, University of Newcastle, Newcastle, Australia. ${ }^{19}$ Department of Respiratory and Sleep Medicine, Hunter Medical Research Institute, John Hunter Hospital, New Lambton Heights, Australia. ${ }^{20}$ Centre for Experimental Medicine, Queen's University Belfast, Belfast, UK. ${ }^{21}$ Alfred Health \& Monash University, Melbourne, Australia. ${ }^{22}$ Department of Respiratory Medicine \& Allergology, Faculty of Medicine, Kindai University Hospital, Ōsakasayama, Japan. ${ }^{23}$ Guy's \& St Thomas' NHS Trust and King's College London, London, UK. ${ }^{24}$ Faculty of Medicine \& Dentistry, University of Plymouth, Plymouth, UK. ${ }^{25}$ Department of Respiratory \& Critical Care Medicine, Singapore General Hospital and Duke-National University Singapore Medical School, Singapore, Singapore. ${ }^{26}$ Allergy Centre, Tampere University Hospital and Tampere University, Tampere, Finland. ${ }^{27}$ Department of Respiratory Medicine, Faculty of Medicine, Landspitali University Hospital and University of Iceland, Reykjavik, Iceland. ${ }^{28}$ University of Amsterdam, Amsterdam, The Netherlands. ${ }^{29}$ Royal Brompton \& Harefield NHS Foundation Trust, London, UK. ${ }^{30}$ University of Athens, Athens, Greece. ${ }^{31}$ University of Manchester, Manchester, UK. ${ }^{32}$ Pneumology Service, Hospital Universitario Lucus Augusti, Lugo, Spain. ${ }^{33}$ University of Sydney Medical School, Sydney, Australia. ${ }^{34}$ UK Severe Asthma Network, Barts Health NHS Trust and Queen Mary University of London, London, UK. ${ }^{35}$ University Hospital "Sv. Ivan Rilski", Sofia, Bulgaria. ${ }^{36}$ Bispebjerg Hospital, Copenhagen University, Copenhagen, Denmark. ${ }^{37}$ The Catholic University of Korea, Seoul, South Korea. ${ }^{38}$ Faculty of Pharmaceutical Sciences, University of British Columbia, Vancouver, Canada. ${ }^{39}$ Division of Allergy \& Clinical Immunology, Department of Medicine, National Jewish Health and Division of Allergy \& Clinical Immunology, Department of Internal Medicine, University of Colorado Hospital, Denver and Aurora, CO, USA. ${ }^{40}$ Division of Pulmonary, Critical Care and Sleep Medicine, Asthma Program, National Jewish Health, Denver, USA. ${ }^{41}$ Observational and Pragmatic Research Institute, Singapore, 
Singapore. ${ }^{42}$ Academic Primary Care, Division of Applied Health Sciences, University of Aberdeen, Polwarth Building, Foresterhill, Aberdeen AB25 2ZD, UK.

Received: 11 December 2019 Accepted: 26 June 2020

Published online: 14 August 2020

\section{References}

1. Lefaudeux D, De Meulder B, Loza MJ, Peffer N, Rowe A, Baribaud F, et al. UBIOPRED clinical adult asthma clusters linked to a subset of sputum omics. J Allergy Clin Immunol. 2017:139:1797-807.

2. Moore WC, Hastie AT, Li X, Li H, Busse WW, Jarjour NN, et al. Sputum neutrophil counts are associated with more severe asthma phenotypes using cluster analysis. J Allergy Clin Immunol. 2014;133:1557-63 e5.

3. Moore WC, Meyers DA, Wenzel SE, Teague WG, Li H, Li X, et al. Identification of asthma phenotypes using cluster analysis in the severe asthma research program. Am J Respir Crit Care Med. 2010;181:315-23.

4. Pavord ID, Beasley R, Agusti A, Anderson GP, Bel E, Brusselle G, et al. After asthma: redefining airways diseases. Lancet. 2018:391:350-400.

5. Chung KF, Wenzel SE, Brozek JL, Bush A, Castro M, Sterk PJ, et al. International ERS/ATS guidelines on definition, evaluation and treatment of severe asthma. Eur Respir J. 2014:43:343-73.

6. Hekking P-PW, Wener RR, Amelink M, Zwinderman AH, Bouvy ML, Bel EH. The prevalence of severe refractory asthma. J Allergy Clin Immunol. 2015; 135:896-902.

7. von Bülow A, Backer V, Bodtger U, Søes-Petersen NU, Vest S, Steffensen I, et al. Differentiation of adult severe asthma from difficult-to-treat asthma outcomes of a systematic assessment protocol. Respir Med. 2018;145:41-7.

8. Chipps BE, Haselkorn T, Paknis B, Ortiz B, Bleecker ER, Kianifard F, et al. More than a decade follow-up in patients with severe or difficult-to-treat asthma: The Epidemiology and Natural History of Asthma: Outcomes and Treatment Regimens (TENOR) II. J Allergy Clin Immunol. 2018;141:1590-7 e9.

9. Dean BB, Calimlim BC, Sacco P, Aguilar D, Maykut R, Tinkelman D. Uncontrolled asthma: assessing quality of life and productivity of children and their caregivers using a cross-sectional internet-based survey. Health Qual Life Outcomes. 2010;8:96.

10. Chastek B, Korrer S, Nagar SP, Albers F, Yancey S, Ortega H, et al. Economic burden of illness among patients with severe asthma in a managed care setting. J Manag Care Spec Pharm. 2016;22:848-61.

11. Sadatsafavi M, Lynd L, Marra C, Carleton B, Tan WC, Sullivan S, et al. Direct health care costs associated with asthma in British Columbia. Can Respir J. 2010;17:74-80

12. Zeiger RS, Schatz M, Dalal AA, Qian L, Chen W, Ngor EW, et al. Utilization and costs of severe uncontrolled asthma in a managed-care setting. J Allergy Clin Immunol Pract. 2016;4:120-9 e3.

13. Heaney LG, Brightling CE, Menzies-Gow A, Stevenson M, Niven RM, British Thoracic Society difficult asthma network. Refractory asthma in the UK: crosssectional findings from a UK multicentre registry. Thorax. 2010:65:787-94.

14. Schleich F, Brusselle G, Louis R, Vandenplas O, Michils A, Pilette C, et al. Heterogeneity of phenotypes in severe asthmatics. The Belgian severe asthma registry (BSAR). Respir Med. 2014;108:1723-32.

15. Korn S, Hübner M, Hamelmann E, Buhl R. The German severe asthma registry. Pneumologie (Stuttgart, Germany). 2012;66:341-4.

16. Heffler E, Blasi F, Latorre M, Menzella F, Paggiaro P, Pelaia G, et al. The severe asthma network in Italy: findings and perspectives. J Allergy Clin Immunol Pract. 2019;7:1462-8.

17. Vennera MDC, Pérez De Llano L, Bardagí S, Ausin P, Sanjuas C, González H et al. Omalizumab therapy in severe asthma: experience from the Spanish registry - some new approaches.J Asthma. 2012:49:416-22

18. Bousquet J, Mantzouranis E, Cruz AA, Aït-Khaled N, Baena-Cagnani CE, Bleecker ER, et al. Uniform definition of asthma severity, control, and exacerbations: document presented for the World Health Organization consultation on severe asthma. J Allergy Clin Immunol. 2010;126:926-38.

19. Global Initiative for Asthma. Global Strategy for Asthma Management and Prevention, 2018. https://www.ginasthma.org. Accessed 13 November 2019.

20. Zervas E, Samitas K, Papaioannou Al, Bakakos P, Loukides S, Gaga M. An algorithmic approach for the treatment of severe uncontrolled asthma. ERJ Open Res. 2018:4:00125-2017.

21. Agache I, Ryan D, Rodriguez MR, Yusuf O, Angier E, Jutel M. Allergy management in primary care across European countries - actual status. Allergy. 2013;68:836-43.
22. Peters DH, Garg A, Bloom G, Walker DG, Brieger WR, Hafizur RM. Poverty and access to health care in developing countries. Ann N Y Acad Sci. 2008; 1136:161-71.

23. European Network of Centres for Pharmacoepidemiology and Pharmacovigilance (ENCePP®). http://www.encepp.eu/encepp/viewResource. htm?id=24389. Accessed 13 November 2019.

24. Optimum Patient Care. The Optimum Patient Care Research Database (OPCRD). http://optimumpatientcare.org/database-overview/. Accessed 13 November 2019.

25. Respiratory effectiveness group (REG). http://effectivenessevaluation.org/. Accessed 13 November 2019

26. Respiratory effectiveness group. ADEPT Committee. http:// effectivenessevaluation.org/adept-committee/. Accessed 13 November 2019.

27. National Asthma Education and Prevention Program, National Heart, Lung and Blood Institute. Expert Panel Report 3: Guidelines for the Diagnosis \& Management of Asthma. 2007. http://www.ncbi.nlm.nih.gov/books/ NBK7232/. Accessed 13 November 2019

28. European Commission. 2018 reform of EU data protection rules. 2018 . https://ec.europa.eu/commission/priorities/justice-and-fundamental-rights/ data-protection/2018-reform-eu-data-protection-rules_en . Accessed 13 Nov 2019

29. SNOMED CT United States Edition. 2019. https:/www.nlm.nih.gov/healthit/ snomedct/us_edition.html. Accessed 13 Nov 2019

30. Okoli C, Pawlowski SD. The Delphi method as a research tool: an example, design considerations and applications. Inf Manag. 2004;42:15-29.

31. Bulathsinhala L, Eleangovan N, Heaney LG, Menzies-Gow A, Gibson PG, Peters $\mathrm{M}$, et al. Development of the International Severe Asthma Registry (ISAR): a modified Delphi study. J Allergy Clin Immunol Pract. 2019;7:578-88 e2.

32. Haldar P, Brightling CE, Hargadon B, Gupta S, Monteiro W, Sousa A, et al. Mepolizumab and exacerbations of refractory eosinophilic asthma. N Engl J Med. 2009;360:973-84.

33. Roufosse FE, Kahn J-E, Gleich GJ, Schwartz LB, Singh AD, Rosenwasser LJ, et al. Long-term safety of mepolizumab for the treatment of hypereosinophilic syndromes. J Allergy Clin Immunol. 2013;131:461-7 e5.

34. Wang E, Wechsler ME, Tran TN, Heaney LG, Jones RC, Menzies-Gow AN, et al. Characterization of severe asthma worldwide: data from the International Severe Asthma Registry. Chest. 2020;157:790-804.

35. Carr TF, Zeki AA, Kraft M. Eosinophilic and noneosinophilic asthma. Am J Resp Crit Care Med. 2018;197:22-37.

36. Bladen CL, Rafferty K, Straub V, Monges S, Moresco A, Dawkins H, et al. The TREAT-NMD Duchenne muscular dystrophy registries: conception, design, and utilization by industry and academia. Hum Mutat. 2013;34:1449-57.

37. Pugliatti M, Eskic D, Mikolcić T, Pitschnau-Michel D, Myhr KM, Sastre-Garriga $J$, et al. Assess, compare and enhance the status of persons with multiple sclerosis (MS) in Europe: a European register for MS. Acta Neurol Scand. 2012;126:24-30

38. Michaud K, Berglind N, Franzén S, Frisell T, Garwood C, Greenberg JD, et al. Can rheumatoid arthritis (RA) registries provide contextual safety data for modern RA clinical trials? The case for mortality and cardiovascular disease. Ann Rheum Dis. 2016;75:1797.

\section{Publisher's Note}

Springer Nature remains neutral with regard to jurisdictional claims in published maps and institutional affiliations.

Ready to submit your research? Choose BMC and benefit from:

- fast, convenient online submission

- thorough peer review by experienced researchers in your field

- rapid publication on acceptance

- support for research data, including large and complex data types

- gold Open Access which fosters wider collaboration and increased citations

- maximum visibility for your research: over $100 \mathrm{M}$ website views per year

At BMC, research is always in progress.

Learn more biomedcentral.com/submissions 